Experimental demonstration of a suspended, diffractively coupled Fabry-Perot cavity

This article has been downloaded from IOPscience. Please scroll down to see the full text article.

2010 Class. Quantum Grav. 27084029

(http://iopscience.iop.org/0264-9381/27/8/084029)

View the table of contents for this issue, or go to the journal homepage for more

Download details:

IP Address: 194.94.224.254

The article was downloaded on 03/06/2010 at 08:39

Please note that terms and conditions apply. 


\title{
Experimental demonstration of a suspended, diffractively coupled Fabry-Perot cavity
}

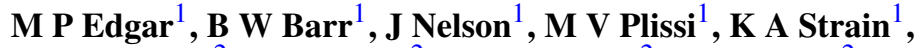 \\ O Burmeister ${ }^{2}$, M Britzger $^{2}$, K Danzmann $^{2}$, R Schnabel ${ }^{2}$, \\ T Clausnitzer ${ }^{3}$, F Brückner ${ }^{3}$, E-B Kley ${ }^{3}$ and A Tünnermann ${ }^{3}$ \\ ${ }^{1}$ Department of Physics and Astronomy, University of Glasgow, Glasgow, G12 8QQ, UK \\ ${ }^{2}$ Max-Planck-Institut für Gravitationsphysik (Albert-Einstein-Institut) and Institut für \\ Gravitationsphysik, Leibniz Universität Hannover, Callinstrasse 38, 30167 Hannover, Germany \\ ${ }^{3}$ Institut für Angewandte Physik, Friedrich-Schiller-Universität Jena, Max-Wien-Platz 1, \\ 07743 Jena, Germany \\ E-mail: m.edgar@physics.gla.ac.uk
}

Received 5 November 2009, in final form 22 January 2010

Published 6 April 2010

Online at stacks.iop.org/CQG/27/084029

\begin{abstract}
Diffraction gratings have been considered as input couplers for Fabry-Perot cavities in future gravitational wave detectors. We experimentally demonstrate the use of a triple-suspended, diffractively coupled cavity and examine conventional Pound-Drever-Hall length sensing and control techniques to maintain the required operating condition. Utilizing the diffractively coupled Fabry-Perot cavity, we investigate the effects associated with translational grating motion and observe a unique $1 / f$ slope in the magnitude of the frequency response when monitoring the forward-reflected error signal.
\end{abstract}

PACS numbers: $04.80 . \mathrm{Nn}, 95.55 . \mathrm{Ym}$

(Some figures in this article are in colour only in the electronic version)

\section{Introduction}

Current laser interferometric gravitational wave detectors (LIGO [1], GEO600 [2], VIRGO [3], and TAMA [4]) operate with partially transmissive components such as beam splitters and cavity input couplers. To enhance detector sensitivity several techniques are implemented, such as power recycling, signal recycling and optical cavities. The power incident on some of the optical components within these Michelson-based topologies is of the order $1 \mathrm{~kW}$ at a wavelength of $1064 \mathrm{~nm}$. Proposed designs for the next generation of gravitational wave detectors require even higher circulating light power to meet ambitious sensitivity targets; however, this has troublesome implications. The optical components are typically manufactured with a fused silica substrate and a dielectric coating. A constant small fraction 
of incident light is always absorbed in the optical substrates of partially transmissive optics, and causes localized heating which leads to thermal lensing due to a change in refractive index with temperature [5]. The strongest thermal lenses that will limit the detector sensitivity will occur inside the beam splitter and cavity input coupler's; hence, investigations into new technologies and interferometer topologies are currently underway to help overcome these concerns.

Diffractive reflection gratings offer a novel approach for splitting and recombining light fields without transmission through optical substrates and is a technology currently being tested for the third generation of detectors like the Einstein Telescope [6]. These devices may also provide the solution for reducing the thermal noise contributions in optical components and suspensions if cryogenic techniques are adopted in the future ${ }^{4}$. For instance, it has been shown that the thermal expansion coefficient of silicon, which is opaque at $1064 \mathrm{~nm}$, can reach zero at low temperatures, suggesting that the thermoelastic noise contribution should also become negligible [7]. There has also been considerable progress towards manufacturing monolithic diffraction gratings, known as waveguide gratings, which could potentially improve thermal noise limits associated with coating materials [8]. However, a theoretical analysis of gratings used as input couplers has revealed additional characteristics to be expected in the detected control signals. In particular, translational motion of the diffractive coupler relative to the laser beam could introduce additional phase and alignment noise when compared to an equivalent traditional cavity configuration [9]. It is therefore important to understand how to model diffractive couplers and validate such simulations by experiment, which henceforth forms the basis of this manuscript.

\section{Upgrading the Glasgow $10 \mathrm{~m}$ prototype}

A number of theoretical and table-top experiments have examined the input-output amplitude and phase relations for a three-port diffractively coupled Fabry-Perot cavity [10, 11]. The Glasgow $10 \mathrm{~m}$ prototype interferometer was commissioned as a diffractively coupled optical cavity, as illustrated in figure 1, for investigating such devices in a suspended environment. The cavity optics in this system were suspended as triple pendulums with two stages of cantilever-mounted spring blades for enhanced vertical isolation and freedom of motion. The diffraction grating under investigation was manufactured by etching a binary structure into a fused silica substrate then coating with multiple alternating layers of tantala $\left(\mathrm{Ta}_{2} \mathrm{O}_{5}\right)$ and silica $\left(\mathrm{SiO}_{2}\right)$ to give an ultra low-loss low-efficiency three-port grating with a period $d=1450 \mathrm{~nm}$ [12]. The grating was mounted in a second-order Littrow configuration and illuminated at an angle of $47.2^{\circ}$ with $181 \mathrm{~mW}$ of $s$-polarized light at $1064 \mathrm{~nm}$ wavelength, from a Nd:YAG laser (Model Mephisto 2000NE from Innolight). The configuration chosen provides weak coupling into and out of the cavity, ensuring that the resulting system is directly comparable with a conventional Fabry-Perot cavity.

\section{Control and length sensing signal extraction}

Conventionally, to maintain a gravitational wave detector at the operating point, monochromatic laser light, known as the carrier light, is held resonant inside the optical cavities of the interferometer by suitable feedback control. Modulation sidebands are added to the carrier light before entering the system, as prescribed in the Pound-Drever-Hall (PDH) technique [13], and the light exiting the cavity is subsequently detected on tuned photodetectors

4 Note: care must be taken to ensure the coating thickness minimizes substrate heating due to evanescent fields. 


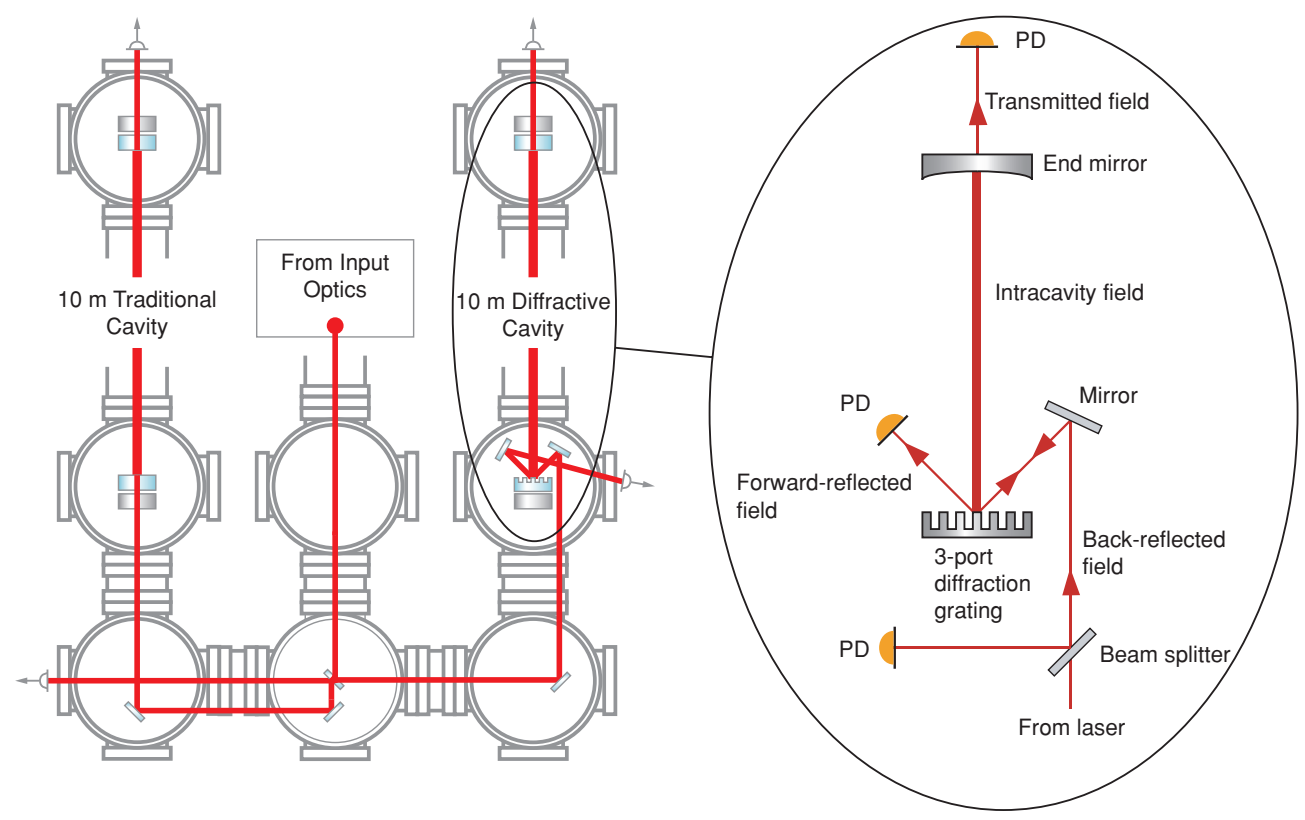

Figure 1. Topology of the Glasgow $10 \mathrm{~m}$ prototype (left) showing the traditional and diffractive interferometer arms and simplified schematic (right) of the three-port grating, used in second-order Littrow configuration, as the input coupler for a diffractive cavity. Tuned photodiodes (PD's) are positioned at all three output ports to detect the dc power and RF component for derivation of the control signals.

located at the output ports. The detected light is demodulated with a local oscillator, at the modulation frequency, to obtain information regarding the interaction of the frequency components. The bi-polar error signal obtained indicates the relative length between the cavity mirrors and can then be used to control the cavity length through suitable electronic feedback. From figure 1 it can be seen that our system has three detection ports, and to enable signal extraction from each output required the carrier light to be twice modulated. As with the traditional PDH technique the modulation frequency for the forward- and back-reflected ports can be arbitrarily chosen and for convenience $10 \mathrm{MHz}$ was used in our system. However, to monitor the cavity length with the transmitted port required the use of sidebands just offresonance at $15.24 \mathrm{MHz}$, close to the free spectral range (FSR) of our cavity of $15.27 \mathrm{MHz}$.

\section{Modelling a diffractively-coupled Fabry-Perot cavity}

The study of interferometer topologies is reasonably straightforward when dealing with the simple relationships among linear light fields, and numerical techniques are routinely used to compute the signals obtained at various photodetectors in response to changes in the relative positions of the optics. This is necessary for the development of length sensing and control schemes required to keep the interferometers at the desired operating point and to read out the signals, including the gravitational wave signal.

A traditional two-port Fabry-Perot cavity couples one input field to two output fields and can be modelled using the amplitude reflection/transmission efficiencies of the mirrors. However, by replacing the input coupler with a three-port diffractive reflection grating, 
Table 1. Parameter values of the grating, provided by AEI in Hannover where it was first tested.

\begin{tabular}{lc}
\hline Parameter & Value $(\%)$ \\
\hline$\rho_{0}^{2}$ & 99.663 \\
$\eta_{0}^{2}$ & 99.840 \\
$\eta_{1}^{2}$ & 0.069 \\
$\eta_{2}^{2}$ & 0.016 \\
\hline
\end{tabular}

each input field couples to three output fields. Implementing the configuration used in this investigation, determines a second-order Littrow incident beam $\left(\theta_{i}=\arcsin (\lambda / d)\right)$ coupling to the orders 0,1 and 2 and a normal incident beam coupling to the orders $-1,0$ and +1 . This type of coupling leads to more complex phase relations compared to a conventional two-port cavity, which can be represented by a scattering matrix:

$$
\mathbf{S}_{3 p}=\left[\begin{array}{lll}
\eta_{2} \mathrm{e}^{\mathrm{i} \phi_{2}} & \eta_{1} \mathrm{e}^{\mathrm{i} \phi_{1}} & \eta_{0} \mathrm{e}^{\mathrm{i} \phi_{0}} \\
\eta_{1} \mathrm{e}^{\mathrm{i} \phi_{1}} & \rho_{0} \mathrm{e}^{\mathrm{i} \phi_{0}} & \eta_{1} \mathrm{e}^{\mathrm{i} \phi_{1}} \\
\eta_{0} \mathrm{e}^{\mathrm{i} \phi_{0}} & \eta_{1} \mathrm{e}^{\mathrm{i} \phi_{1}} & \eta_{2} \mathrm{e}^{\mathrm{i} \phi_{2}}
\end{array}\right],
$$

where $\eta_{0,1,2}$ and $\phi_{0,1,2}$ are the amplitude diffraction efficiencies and phase changes on diffraction for zeroth, first and second orders respectively, and $\rho_{0}$ is the amplitude reflectivity at normal incidence. For each output port of the cavity, the field amplitudes have already been investigated [10] and can be described by

$$
\begin{aligned}
& c_{1}=\eta_{2} \mathrm{e}^{\mathrm{i} \phi_{2}}+\eta_{1}^{2} \mathrm{e}^{2 \mathrm{i}\left(\phi_{1}+\phi\right)} d, \\
& c_{2 t}=\mathrm{i} \tau_{1} \eta_{1} \mathrm{e}^{\mathrm{i}\left(\phi_{1}+\phi\right)} d, \\
& c_{3}=\eta_{0}+\eta_{1}^{2} \mathrm{e}^{2 \mathrm{i}\left(\phi_{1}+\phi\right)} d,
\end{aligned}
$$

where the resonance factor is defined by $d=\left[1-\rho_{0} \rho_{1} \mathrm{e}^{2 \mathrm{i} \phi}\right]^{-1}, \phi$ represents the phase acquired after one round trip of the cavity, and $\rho_{1}$ and $\tau_{1}$ are the amplitude reflectivity/transmittivity efficiencies of the end mirror. From the specifications of our grating, as presented in table 1, and the reflection/transmission efficiencies $\left(\rho_{1} / \tau_{1}\right)$ of the end mirror, all the cavity properties can be determined numerically. Furthermore, based on the field equations (2)-(4) a numerical simulation of the diffractive cavity could be built, using the MATLAB software package. This simulation allowed us to compare experimental findings for the amplitude and power of output light fields, with modelled predictions.

\section{Experimental results}

The FSR of our cavity is $15.27 \mathrm{MHz}$ and by monitoring the dc signal from the transmitted port, the corresponding full width at half-maximum (FWHM) was determined to be $13.80 \pm 0.64 \mathrm{kHz}$. The finesse of our cavity was measured to be $1107 \pm 51$, and from the simulation an expected finesse of the diffractive cavity was calculated to be $1177 \pm 27$, showing good agreement. From the grating parameters used in our model, we were also able to calculate the expected grating loss using the identity $L_{G}=1-\left(\rho_{0}^{2}+2 \eta_{1}^{2}\right)$, which was determined to be $0.199 \%$, closely matching a measured value of $0.177 \% \pm 0.025 \%$ carried out at AEI in Hannover. Furthermore, based on the specifications of the end mirror and the measured dc light power detected at the transmitted output port, we were able to calculate the circulating power in the cavity to be around $15 \mathrm{~W}$. 

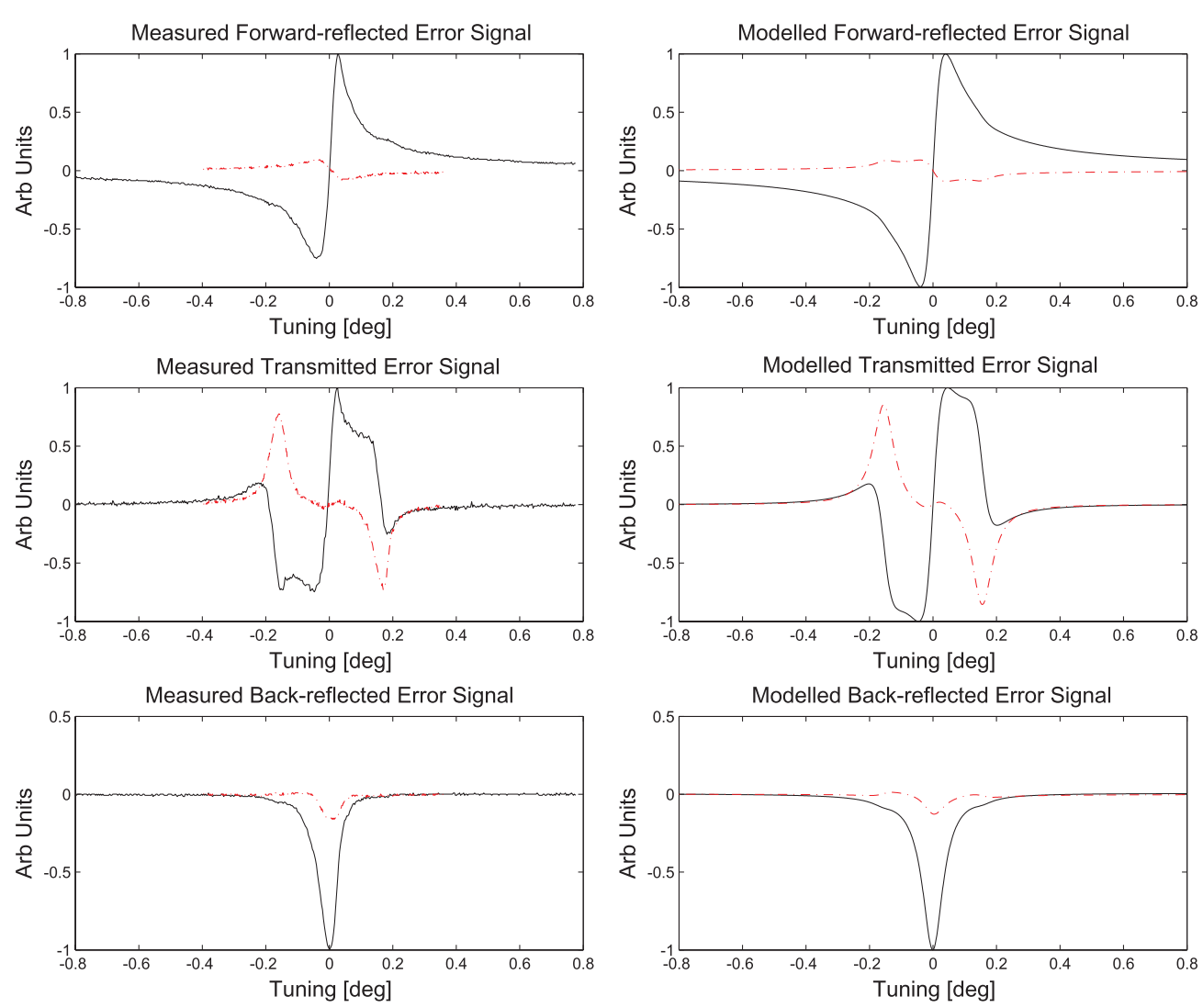

Figure 2. Normalized RF power for each output port; forward-reflected (top), transmitted (middle), and back-reflected (bottom). The measured data and modelled predictions of the length sensing signals are presented on the left- and right-hand sides respectively. The solid (black) trace indicates in-phase measurements, and the dashed (red) trace indicates quadrature-phase. The absolute scaling between modelled forward-reflected, transmitted, and back-reflected ports is 163:1:2.

One way to establish whether the sensing signals predicted by the model agree with experiment is to sweep the length of the cavity by at least one round-trip wavelength (one FSR in frequency terms). Then the demodulated signal from each port can be compared to the prediction. The slope of this signal at the operating point gives the response for that output port, otherwise known as the effective optical gain. In this experiment, instead of sweeping the cavity length the laser frequency was swept. This has the advantage that it does not risk causing alignment changes of the cavity that could cause higher order cavity modes to become excited thus producing extra features in the result.

The radio-frequency $(\mathrm{RF})$ signal from each detection port can be demodulated in two orthogonal phases, we call these 'in-phase' and 'quadrature-phase' respectively. The demodulation phase in the 'quadrature-phase' case was adjusted to minimize the detected signal, while the 'in-phase' signal was obtained by adding a $90^{\circ}$ phase shift in the demodulation process. The demodulated signals obtained in the experiment are shown in figure 2 and are seen to be in good qualitative agreement with modelled predictions. The discrepancies between the experiment and modelled length sensing signals can be attributed to the finite rate of sweep in the experiment. The model is 'quasi-static' and therefore does not predict the slight asymmetry in the patterns seen in the experiment as stored light leaks out after a small delay. 
Table 2. The measured and modelled signal response for each port shows the correct scaling relative to transmitted port.

\begin{tabular}{lcc}
\hline Parameter & Measured value $(\mathrm{dB})$ & Modelled value $(\mathrm{dB})$ \\
\hline Back-reflected/transmitted & -26.93 & -22.51 \\
Transmitted/transmitted & 0 & 0 \\
Forward-reflected/transmitted & 43.07 & 44.74 \\
\hline
\end{tabular}

There is a practical limit to how slow a sweep can be made due to uncontrolled $1 \mathrm{~Hz}$ motion of the pendulums, since longitudinal, lateral and angular motions of the mirrors cause small misalignments. These effects are almost unavoidable with suspended optics.

It is essential to calibrate the demodulated signals detected at each port with the modelled signals to investigate the level of quantitative agreement. This required comparison between the relative size of the in-phase slopes to that of the transmitted port. The ratios of the signal responses are presented in table 2 and indicate good agreement.

Analysis of figure 2 indicates that only the transmitted signal will be symmetrical around the centre of resonance. This is because light has been diffracted into the cavity only once, and therefore all frequency components receive the same phase shift before resonating. From previous investigations with table-top cavities [11], the extent of the asymmetry was seen to be determined by the values of the $\eta_{0}$ and $\eta_{2}$ diffraction efficiencies. With our numerical simulation accurately validated by experiment, we were also able to probe the effects of asymmetry on the demodulated output signals by altering the grating parameters. An interesting result of this analysis is that, through a careful choice of demodulation phase, we can extract signals from each of the reflected ports which sum together to reconstruct a traditional PDH locking signal (see figure 3). This has been compared to other measurements carried out with different apparatus that show the same effect, made at AEI [14].

The diffractive optic used in our investigation had a second-order diffraction efficiency close to the minimum possible; hence, the forward-reflected signal had a shape that closely resembled the PDH signal. It was possible to lock the cavity using the signal from this port, although not quite at the centre of resonance; therefore, the cavity was instead locked to the transmitted error signal (which does exhibit symmetrical behaviour around the centre of resonance). Locking to this port also makes it possible to investigate the dynamic behaviour and additional effects associated with suspended diffractively coupled optical systems.

Investigations of grating interferometers have shown additional phase and alignment noise, when compared to traditional mirror topologies [9]. Utilizing electromagnetic actuators positioned at the rear and at the side of the test mass, which holds the diffractive optic, it was possible to inject motion along the cavity axis (longitudinal) and also perpendicular to the cavity axis (translational). By observing the forward-reflected signal, we were able to demonstrate the effects associated with translational grating motion. Injecting longitudinal motion on the test mass produces a $1 / f^{2}$ shape in the magnitude of the frequency response of the demodulated signal. This effect is seen in both mirror coupled and diffractively coupled cavities. However, translational motion of the grating produces a $1 / f$ shape which is not observed in a mirror-coupled cavity and can therefore be attributed to the properties of the grating. A full theoretical analysis has been undertaken by our colleagues at Birmingham University and has now been published [15]. This new feature is due to side induced phase sidebands, introduced on the light entering the cavity, that propagate through the cavity and then interact with additional side induced phase sidebands exiting the cavity. Many of the frequency components on the exiting light cancel; however, the remainder can be detected on 


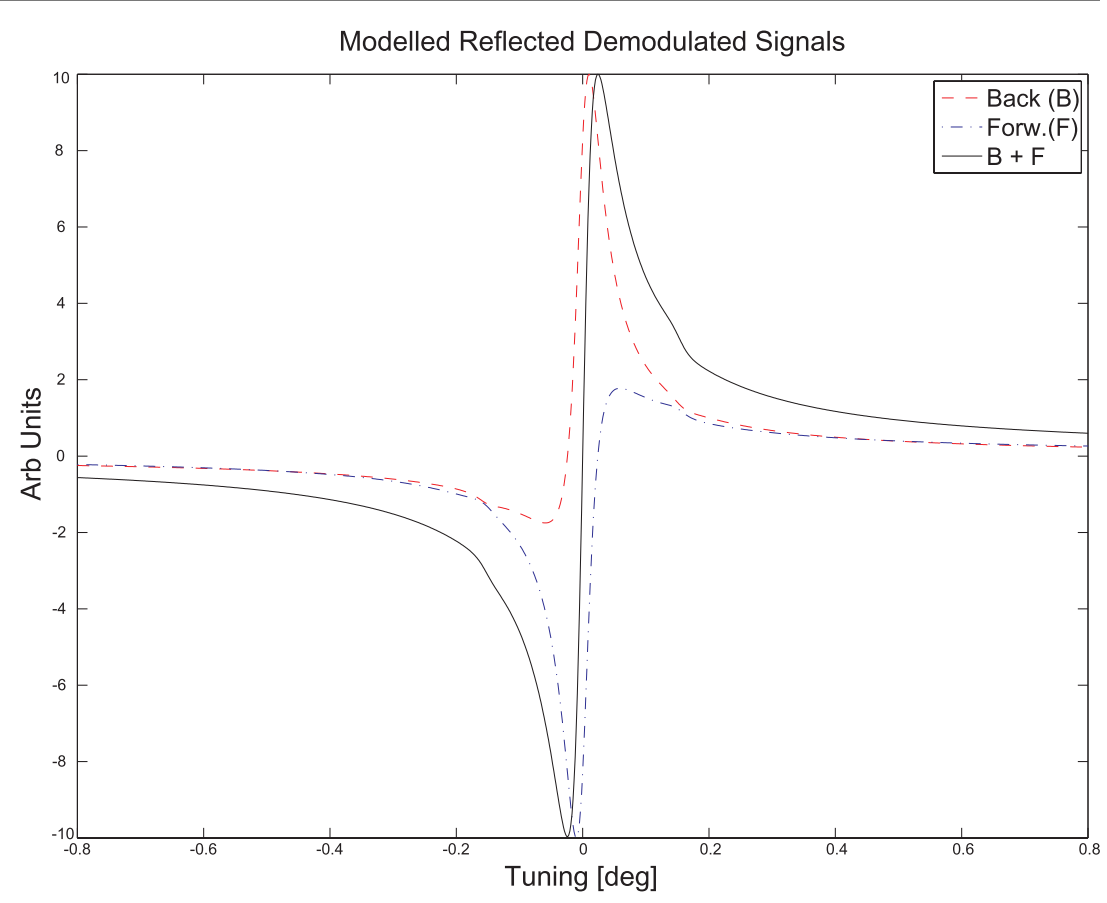

Figure 3. Modelled demodulated signals; back-reflected (red) and forward-reflected (blue) indicated by dashed traces, and the combined back-reflected + forward-reflected (black) indicated by a solid trace. The grating parameters used here are for an ideal (lossless) grating with $\rho_{0}=0.99663, \eta_{1}=0.0407$ and $\eta_{0}=\eta_{2}=0.7065$.

a photodetector and demodulated to give this unique behaviour. A full analysis of this work has been carried out and is in the process of publication.

\section{Conclusion}

In conclusion, we have constructed a suspended diffractively coupled Fabry-Perot cavity within the Glasgow $10 \mathrm{~m}$ prototype interferometer and developed a numerical model for the system. From the simulation we were able to investigate the use of conventional techniques for length sensing and control signal extraction from a diffractively coupled Fabry-Perot cavity. Our experimental results provided qualitative and quantitative verification of the theoretical framework supporting grating interferometers. It was possible to adapt our numerical model to show the extent of asymmetry in both reflected demodulated signals and furthermore reconstruct a symmetrical PDH signal with these signals, irrespective of their shape. Additionally, we have revealed the unique behaviour associated with translational grating motion and verified the $1 / f$ shape in the frequency response with theoretical predictions. Further work on the dynamic effects associated with gratings will be published in a later paper.

\section{Acknowledgments}

The authors would like to thank Szabi and Zsuzsa Marka and all other organizers of the Amaldi8 conference in New York. This work was supported by the University of Glasgow 
and the Science and Technology Facilities Council. The authors would also like to thank the Sonderforschungsbereich TR7 of the Deutsche Forschungsgemeinschaft for financial support.

\section{References}

[1] Sigg D 2002 Class. Quantum Grav. 19 1429-35

[2] Willke B et al 2002 Class. Quantum Grav. 19 1377-87

[3] Acernese F et al 2002 Class. Quantum Grav. 19 1421-8

[4] Ando M 2002 and the TAMA collaboration Class. Quantum Grav. 19 1409-19

[5] Strain K A, Danzmann K, Mizuno J, Nelson P G, Rüdiger A, Schilling R and Winkler W 1994 Phys. Lett. A $194124-32$

[6] Punturo M et al 2010 Class. Quantum Grav. 27084007

[7] Rowan S, Hough J and Crooks D R M 2005 Phys. Lett. A 347 25-32

[8] Brückner F, Clausnitzer T, Burmeister O, Friedrich D, Kley E-B, Danzmann K, Tünnermann A and Schnabel R 2008 Opt. Lett. 33 264-6

[9] Freise A, Bunkowski A and Schnabel R 2007 New J. Phys. 9433

[10] Bunkowski A, Burmeister O, Danzmann K and Schnabel R 2005 Opt. Lett. $301183-5$

[11] Bunkowski A, Burmeister O, Danzmann K, Schnabel R, Clausnitzer T, Kley E-B and Tünnermann A 2006 Opt. Lett. 31 2384-6

[12] Clausnitzer T, Kley E-B, Tünnermann A, Bunkowski A, Burmeister O, Danzmann K, Schnabel R, Gliech S and Duparrè A 2005 Opt. Express 13 4370-8

[13] Drever R W P, Hall J L, Kowalski F V, Hough J, Ford G M, Munley A J and Ward H 1983 Appl. Phys. B 31 97-105

[14] Britzger M 2008 private communication

[15] Hallam J, Chelkowski S, Freise A, Hild S, Barr B, Strain K A, Burmeister O and Schnabel R 2009 J. Opt. A: Pure Appl. Opt. 11085502 